\title{
Spectroscopy of a one-dimensional V-shaped quantum well with a point impurity
}

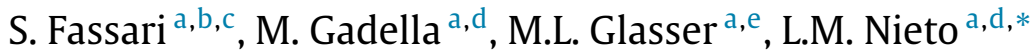 \\ a Departamento de Física Teórica, Atómica y Óptica, Universidad de Valladolid, 47011 Valladolid, Spain \\ b CERFIM, PO Box 1132, Via F. Rusca 1, CH-6601 Locarno, Switzerland \\ c Dipartimento di Fisica Nucleare, Subnucleare e delle Radiazioni, Universitá degli Studi Guglielmo Marconi, \\ Via Plinio 44, I-00193 Rome, Italy \\ d IMUVA-Instituto de Matemáticas, Universidad de Valladolid, 47011 Valladolid, Spain \\ e Department of Physics, Clarkson University, Potsdam, NY 13699, USA
}

\section{A R T I C L E I N F O}

\section{Article history:}

Received 1 August 2017

Accepted 14 December 2017

Available online 18 December 2017

\section{Keywords:}

V-shaped potential

Point interaction

Level crossing

Level rearrangement

\begin{abstract}
A B S T R A C T
We consider the one-dimensional Hamiltonian with a V-shaped potential $H_{0}=\frac{1}{2}\left[-\frac{d^{2}}{d x^{2}}+|x|\right]$, decorated with a point impurity of either $\delta$-type, or local $\delta^{\prime}$-type or even nonlocal $\delta^{\prime}$-type, thus yielding three exactly solvable models. We analyse the behaviour of the change in the energy levels when an interaction of the type $-\lambda \delta(x)$ or $-\lambda \delta\left(x-x_{0}\right)$ is switched on. In the first case, even energy levels, pertaining to antisymmetric bound states, remain invariant with respect to $\lambda$ even though odd energy levels, pertaining to symmetric bound states, decrease as $\lambda$ increases. In the second, all energy levels decrease when the factor $\lambda$ increases. A similar study has been performed for the so-called nonlocal $\delta^{\prime}$ interaction, requiring a coupling constant renormalisation, which implies the replacement of the form factor $\lambda$ by a renormalised form factor $\beta$. In terms of $\beta$, odd energy levels are unchanged. However, we show the existence of level crossings: after a fixed value of $\beta$ the energy of each even level, with the natural exception of the first one, becomes lower than the constant energy of the previous odd level. Finally, we consider an interaction of the type $-\lambda \delta(x)+\mu \delta^{\prime}(x)$, and analyse in detail the discrete spectrum of the resulting selfadjoint Hamiltonian.
\end{abstract}

(C) 2017 Elsevier Inc. All rights reserved.

\footnotetext{
* Corresponding author at: Departamento de Física Teórica, Atómica y Óptica, Universidad de Valladolid, 47011 Valladolid, Spain.

E-mail address: luismiguel.nieto.calzada@uva.es (L.M. Nieto).
} 


\section{Introduction}

One-dimensional point potentials in quantum mechanics [1,2] and in quantum field theory (QFT) have received a lot of attention in the past two decades. They serve to model realistic physical situations with a number of practical applications. They are used to model several kinds of extra thin structures [3,4] or to model point defects in materials, so that effects like tunnelling are easily studied. They are also used in the study of heterostructures, where they may appear in connection with an abrupt effective mass change [5]. In addition, they constitute a class of solvable or quasi-solvable potentials suitable to study basic quantum properties, stationary states, scattering, resonances, etc, where one uses point interactions of the form $\delta$ or $\delta^{\prime}$ or a linear combination thereof [6-17]. A rigorous definition of perturbations of the form $-a \delta(x)+b \delta^{\prime}(x), a$ and $b$ being real numbers, is rather technical and we are not going to deal with this here.

Given the increasing relevance of one-dimensional quantum dots in the nanophysics literature [18-20], point potentials can be used to model sharply peaked impurities inside the dot. In other physical contexts like scalar QFT on a line, point potentials serve to model impurities and provide external singular backgrounds where the bosons move [21]. The spectra of Hamiltonians with $\delta$ and $\delta^{\prime}$ point interactions provide one-particle states in scalar $(1+1)$-dimensional QFT systems [22-24]. In particular, configurations of two pure delta potentials added to the free Schrödinger Hamiltonian have been used to describe scalar field fluctuations on external backgrounds [25], in terms of the corresponding scattering waves. Delta point interactions allow the implementation of some boundary conditions compatible with a scalar QFT defined on an interval [26]. The delta interaction is often multiplied by a real number $a$. In particular, this coupling of $a$ to the $\delta$ potential mathematically describes the plasma frequencies in Barton's hydrodynamical model [27], characterising the electromagnetic properties of infinitely thin conducting plates. On the other hand, the physical meaning of the $b$ coupling to the $\delta^{\prime}$-interaction in the case of an interaction term of the form $b \delta^{\prime}$ (with the prime denoting the derivative of the delta in the sense of distributions), appearing in the context of Casimir physics, has only recently been interpreted [28]: it describes the response of the orthogonal polarisability of a mono-atomically thin plate to the electromagnetic field.

There are other physical reasons to consider the one-dimensional harmonic oscillator perturbed by point potentials, particularly in the theory of Bose-Einstein condensates [29-31]. It has been very widely studied in the mathematical physics literature [10,32-39] including the three-dimensional case [40-43].

The objective of this paper is the study of the energy levels of the one-dimensional Hamiltonian

$$
\tilde{H}=\tilde{H}_{0}+\tilde{V}:=-\frac{\hbar^{2}}{2 m} \frac{d^{2}}{d y^{2}}+\gamma|y|+\tilde{V}(y), \quad \gamma>0,
$$

where $\tilde{V}(y)$ is a point perturbation of one of the following types:

(i) $\tilde{V}(y)=-a \delta\left(y-y_{0}\right)$, a Dirac distribution centred at any point,

(ii) $\tilde{V}(y)=-a \delta(y)+b \delta^{\prime}(y)$, a combination of a Dirac distribution centred at the origin with its derivative multiplied by a different coupling parameter so that the special cases with either $a=0$ or $b=0$ may be considered,

(iii) $\tilde{V}(y)=-c\left|\delta^{\prime}(y)\right\rangle\left\langle\delta^{\prime}(y)\right|$, a nonlocal $\delta^{\prime}$-interaction.

The denomination V-shaped potential comes, rather obviously, from the shape of the potential $|y|$. Although the $\mathrm{V}$-shaped potential is not qualitatively different from the harmonic one, or any similar confining potential for that matter, we believe it deserves to be investigated in detail given its relevance in the theory of $\delta$-doped quantum wells (materials inside which electrons can move freely in two dimensions but are confined in the third one [44]), as attested by the extent of the relevant literature [45-50]. The combination of the V-shaped potential $\gamma|y|$ with $\tilde{V}(y)$ leads to a singular funnel shaped potential. We remind the reader that, although the term "funnel potential" (or "funnel interaction") was coined in meson physics with reference to the central potential $V(r)=a r-b / r$ in three dimensions (see [51-53]), it has later been used, referring to different potentials, in other 
areas of physics (see [54-56]). It is noteworthy that while there is no ambiguity as to the meaning of the $\delta$-interaction, there have been different interpretations of the meaning of the $\delta^{\prime}$-interaction in the literature on the subject (see [57] for a thorough critical review of the various interpretations found in the relevant literature). In this paper, we are going to compare the results, especially from the spectral point of view, obtained with the two different choices of $\delta^{\prime}$ mentioned above.

The quest for remarkable spectral properties of the total Hamiltonian $\tilde{H}$ (in particular, the dependence of the energy levels on the parameters) requires the use of some rescaling in order to simplify the expressions. We start by setting $y=q x$ where $q$ is a positive real number to be determined next. Then, the Hamiltonian is given by:

$$
\tilde{H}=-\frac{\hbar^{2}}{2 m q^{2}} \frac{d^{2}}{d x^{2}}+\gamma q|x|-\frac{a}{q} \delta\left(x-x_{0}\right),
$$

in case (i);

$$
\tilde{H}=-\frac{\hbar^{2}}{2 m q^{2}} \frac{d^{2}}{d x^{2}}+\gamma q|x|-\frac{a}{q} \delta(x)+\frac{b}{q^{2}} \delta^{\prime}(x)
$$

in case (ii);

$$
\tilde{H}=-\frac{\hbar^{2}}{2 m q^{2}} \frac{d^{2}}{d x^{2}}+\gamma q|x|-\frac{c}{q^{4}}\left|\delta^{\prime}(x)\right\rangle\left\langle\delta^{\prime}(x)\right|,
$$

in case (iii).

After setting $H=\tilde{H} /(2 \gamma q)$ and choosing $q^{3}=\hbar^{2} /(2 m \gamma)$, the new Hamiltonian reads:

$$
H=H_{0}+V=\frac{1}{2}\left[-\frac{d^{2}}{d x^{2}}+|x|\right]+V(x),
$$

with

$$
V(x)=-a\left(\frac{m^{2}}{2 \gamma \hbar^{4}}\right)^{\frac{1}{3}} \delta\left(x-x_{0}\right)=-\lambda \delta\left(x-x_{0}\right),
$$

in case (i),

$$
V(x)=-a\left(\frac{m^{2}}{2 \gamma \hbar^{4}}\right)^{\frac{1}{3}} \delta(x)+\frac{m b}{\hbar^{2}} \delta^{\prime}(x)=-\lambda \delta(x)+\mu \delta^{\prime}(x),
$$

in case (ii), and

$$
V(x)=-c\left(\frac{4 \gamma^{2} m^{5}}{\hbar^{10}}\right)^{\frac{1}{3}}\left|\delta^{\prime}(y)\right\rangle\left\langle\delta^{\prime}(y)|=-\beta| \delta^{\prime}(x)\right\rangle\left\langle\delta^{\prime}(x)\right|,
$$

in case (iii).

In determining the spectral properties of our model, we shall use a technique that relies on some properties of Green's functions. This idea has precedents in the study of quantum dots and quantum wires, as for instance in [58-60], where Coulomb impurities are replaced by nonlocal interactions instead of point interactions as is our case. This method has the additional advantage of relying on a rigorous way of achieving a self-adjoint determination of (5) based on the operator resolvent and the Krein formula [2]. As previously stated, this paper is not the right place to discuss these mathematical subtleties.

In the present manuscript, we begin by considering the Green function for the free Hamiltonian, $H_{0}$, as in (5). This operator can be easily written as an expansion in terms of its eigenfunctions and eigenvalues involving the Airy function, its derivative and their zeros, as a consequence of results given in [61]. Then, we add the delta interaction $V(x)=-\lambda \delta\left(x-x_{0}\right)$.

The coupling constant, $\lambda$, multiplying the $\delta$-interaction will always be preceded by the minus sign throughout this article. The reason lies in its convenience in showing the full manifestation of the 
so-called Zeldovich effect, also known as level rearrangement, present in various models considered in the literature $[10,42,62,63]$ and one of the objectives of our study.

It is not difficult to obtain the Green function of the total Hamiltonian including the deltas. We obtain bound states as solutions of a transcendental equation involving the Airy function and its derivative. Then, we study the inverse problem, i.e., being given two energy values, and assuming the potential to be $\frac{1}{2}|x|-\lambda \delta\left(x-x_{0}\right)$, find the precise values of $\lambda$ and $x_{0}$. With this generality, the problem does not have a unique solution in contrast to the situation analysed in [33], where only a local analysis is carried out for the two lowest eigenvalues.

The next step consists in the decoration of $H_{0}$ with a point potential involving the derivative of the Dirac distribution. Here, there are at least two non-equivalent possibilities, which we shall consider in this article, both leading to the determination of a self-adjoint operator. Both options can be given in terms of matching conditions that wave functions must fulfil at the origin. Let us just say in passing that these matching conditions determine different domains for $H_{0}$ and, therefore, different self-adjoint extensions of $H_{0}$, as defined on a restricted dense domain.

The former, that we call the $\left|\delta^{\prime}\right\rangle\left\langle\delta^{\prime}\right|$ interaction, has been considered by several authors $[1,11,12,64]$ and is determined by the matching conditions: if $\psi(x)$ is the wave function, its derivative $\psi^{\prime}(x)$ is continuous at the origin but $\psi(x)$ itself satisfies the condition $\psi\left(0^{+}\right)-\psi\left(0^{-}\right)=-\beta \psi^{\prime}(0)$, where $\beta$ is a fixed real number, i.e., the coefficient of the $\delta^{\prime}$-interaction. By using the standard matrix notation, the above condition can be written as:

$$
\left(\begin{array}{c}
\psi\left(0^{+}\right) \\
\psi^{\prime}\left(0^{+}\right)
\end{array}\right)=\left(\begin{array}{cc}
1 & -\beta \\
0 & 1
\end{array}\right)\left(\begin{array}{l}
\psi\left(0^{-}\right) \\
\psi^{\prime}\left(0^{-}\right)
\end{array}\right)
$$

In this case, a naive calculation of the Green's function shows divergencies, so that a renormalisation is necessary. We first introduce an ultraviolet energy cut-off and then the ensuing renormalisation of the coupling constant. Renormalisation procedures are necessary for point potentials not only in higher dimensions [1,2], but also in one-dimensional problems when the kinetic energy operator is proportional to the magnitude of the momentum and not to its square, as in the relativistic case [6-8]. Again, eigenvalues are obtained through a transcendental equation involving the Airy function and its derivative. The obtained results are far more interesting than those for the $\delta$-interaction. In particular, the odd energy levels are not affected by the interaction. Note that here these odd energy levels correspond to symmetric bound states, in opposition to what is customary for the harmonic oscillator. On the other hand, even energy levels are strongly affected. They correspond to antisymmetric bound states. For negative values of the coefficient $\beta$, their energies are kept higher than the energy of the next lower odd level. However, after $\beta=0$ the energy decreases sharply and for a certain value of $\beta=\beta_{0}$, which is the same in all cases, $\beta_{0} \approx 1.37172$, the energy of each even energy level coincides with the energy of the next lower odd level. For values $\beta>\beta_{0}$, the energy of the even level decreases further, so that we are in the presence of a quantum phase transition.

A second $\delta^{\prime}$ interaction, which is compatible with the $\delta$ interaction, so that we may compose interactions of the form $V(x)=-\lambda \delta(x)+\mu \delta^{\prime}(x)$ [65-67], comes from the consideration of the following matching conditions for the wave function at the origin:

$$
\left(\begin{array}{c}
\psi\left(0^{+}\right) \\
\psi^{\prime}\left(0^{+}\right)
\end{array}\right)=\left(\begin{array}{cc}
\frac{1+\mu}{1-\mu} & 0 \\
\frac{2 \lambda}{1-\mu^{2}} & \frac{1-\mu}{1+\mu}
\end{array}\right)\left(\begin{array}{c}
\psi\left(0^{-}\right) \\
\psi^{\prime}\left(0^{-}\right)
\end{array}\right) .
$$

The problem is explicitly solved and our findings show that, if the potential is purely of $\delta^{\prime}$ type $(\lambda=0)$, there are no new eigenvalues. On the other hand, if the potential is purely of $\delta$ type $(\mu=0)$, we get the results of Section 3.

\section{The spectrum of $H_{0}$ perturbed by an attractive $\delta$-interaction}

Let us consider $H_{0}$, the Hamiltonian with a V-shaped confinement. Its spectral properties have been investigated in [61]. In particular, we know that it has a complete set of eigenvectors, $\psi_{n}$, with 
eigenvalues $E_{n}, n=1,2,3, \ldots$ Consequently, it admits the following spectral decomposition:

$$
H_{0}=\frac{1}{2}\left[-\frac{d^{2}}{d x^{2}}+|x|\right]=\sum_{n=1}^{\infty} E_{n}\left|\psi_{n}\right\rangle\left\langle\psi_{n}\right| .
$$

Using the above input and (3.8) in [68], we can obtain its Green function (integral kernel of the resolvent), which is

$$
\begin{aligned}
G_{0}(x, y ; E) & =\sum_{n=1}^{\infty} \frac{\psi_{n}(x) \psi_{n}(y)}{E_{n}-E}=-\frac{A i\left(x_{>}-2 E\right) A i\left(-x_{<}-2 E\right)}{A i(-2 E) A i^{\prime}(-2 E)} \\
& =-\frac{A i\left(\frac{x+y+|x-y|}{2}-2 E\right) A i\left(\frac{-x-y+|x-y|}{2}-2 E\right)}{A i(-2 E) A i^{\prime}(-2 E)},
\end{aligned}
$$

where $A i(y)$ is the Airy function and $A i^{\prime}(y)$ its derivative with respect to the variable $y$.

Although this remark has been already included in the Introduction, let us say again that the eigenvalues and the eigenfunctions of the symmetric (respectively antisymmetric) bound states are labelled by an odd (even) index, in contrast to the traditional labelling used for the harmonic oscillator.

As is well known, the eigenvalues of a self-adjoint operator are the zeros of the denominator of the Green function. In our case, we have the operator $H_{\lambda, x_{0}}:=H_{0}-\lambda \delta\left(x-x_{0}\right)$, where $x_{0}$ is any fixed real number. Now, one needs to use sophisticated mathematical tools, such us the Krein formula [2], in order to determine the poles of the Green function. The detailed procedure is given in [69]. Then, since the explicit formula for the Green's function of $H_{\lambda, x_{0}}$ is:

$$
G_{\lambda, x_{0}}(x, y ; E)=G_{0}(x, y ; E)+\frac{G_{0}\left(x, x_{0} ; E\right) G_{0}\left(x_{0}, y ; E\right)}{\frac{1}{\lambda}-G_{0}\left(x_{0}, x_{0} ; E\right)},
$$

in terms of the Green function $G_{0}(x, y ; E)$ given in (12).

Thus, for a fixed value of the positive coefficient $\lambda$, bound states, that is the poles of the Green function (13), are determined by the following transcendental equation:

$$
\lambda=-\frac{A i(-2 E) A i^{\prime}(-2 E)}{\left.\operatorname{Ai(} x_{0}-2 E\right) A i\left(-x_{0}-2 E\right)} .
$$

To begin with, let us choose $x_{0}=0$ in (14), so that it reads,

$$
\lambda=-\frac{A i^{\prime}(-2 E)}{A i(-2 E)} \text {. }
$$

The right hand side of the above equation is a multivalued function of the energy $E$ that we may represent as $\lambda\left(E, x_{0}=0\right)$. Thus, the $n$th eigenvalue is determined by the intersection between the $n$th branch of the function and the horizontal line provided by the value of the coupling constant $\lambda$. We show this in Fig. 1, where we have chosen $\lambda=2$.

The multivalued function $\lambda\left(E, x_{0}=0\right)$ has infinitely many branches, each of which is a strictly decreasing function of the energy parameter. Then, the inverse function of the $n$th branch is the $(2 n-1)$-th eigenenergy $E_{2 n-1}\left(\lambda, x_{0}=0\right)$. The eigenvalues corresponding to the antisymmetric bound states are not modified by the perturbation. Thus for any real $\lambda$, we have that $E_{2 n}\left(\lambda, x_{0}=0\right)=$ $E_{2 n}\left(0, x_{0}=0\right)=E_{2 n}$. Here, $E_{2 n}$ are the even energy values obtained as poles of both (12) and (14), that is to say as solutions of $A i(-2 E)=0$.

In Fig. 2, we plot the five lowest energy eigenvalues as functions of the coupling constant $\lambda$. The eigenvalues of the two lowest antisymmetric bound states, namely $E_{2}\left(0, x_{0}=0\right), E_{4}\left(0, x_{0}=0\right)$, are the horizontal asymptotes of the functions $E_{2 n+1}\left(0, x_{0}=0\right)$ corresponding to the symmetric bound states.

There are two important differences between our Fig. 2 and Figure 1 in [15]. First of all, the unperturbed operator in [15] is twice our free Hamiltonian, $H_{0}$. Moreover, in our total Hamiltonian $H_{\lambda, x_{0}}$, we have written a minus sign in front of $\lambda$. This makes the interaction more attractive as we move from the left to the right along the horizontal axis. We have chosen this option, since we wish to show the manifestation of the Zeldovich effect, also called level rearrangement, in this model. This effect 
$\lambda$

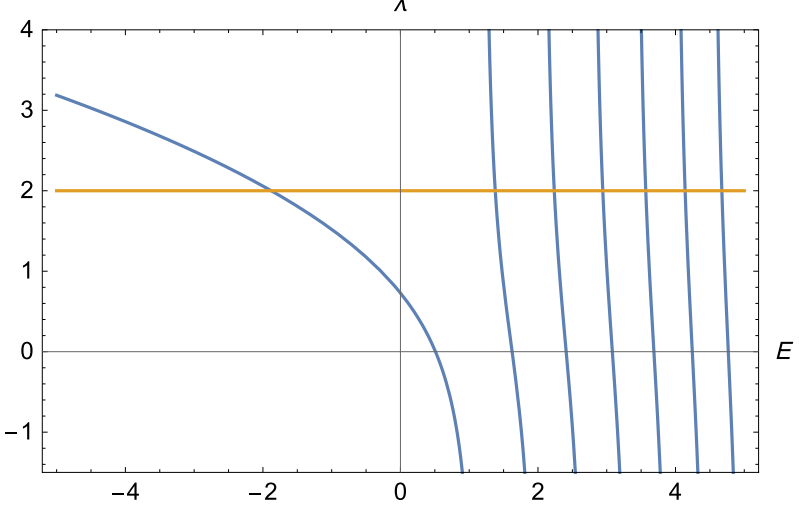

Fig. 1. The $n$th eigenvalue of $H_{\lambda=2, x_{0}=0}$ is given by the intersection between the $n$th branch of the right hand side of (14) as a function of the energy and the horizontal line $\lambda=2$.

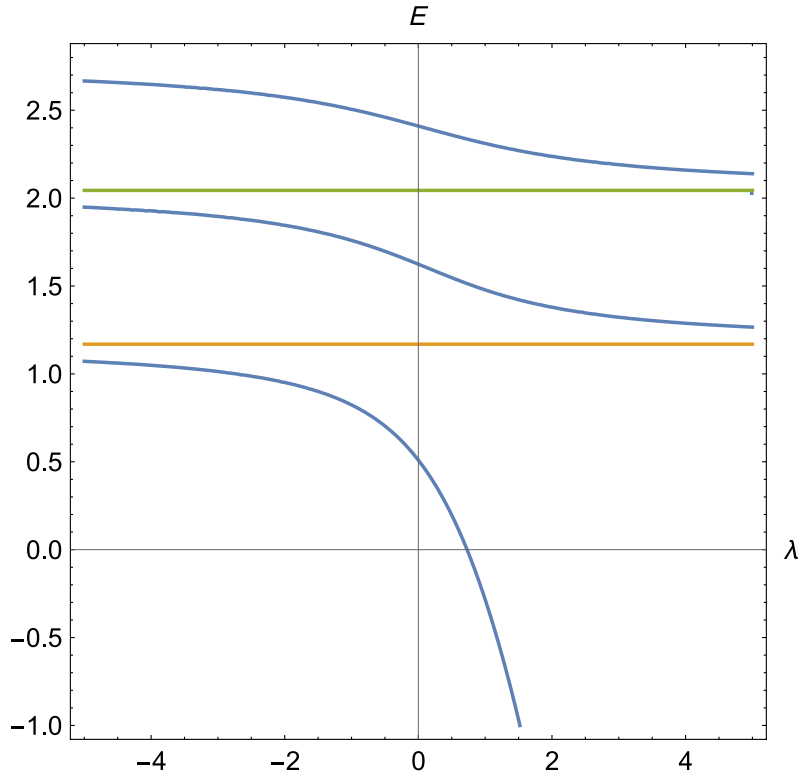

Fig. 2. Plot of the five lowest eigenenergies $E_{n}\left(\lambda, x_{0}=0\right)$ coming from (14). The even eigenvalues do not depend on $\lambda$ : $E_{2}(\lambda, 0)=E_{2}(0,0)$ in yellow and $E_{4}(\lambda, 0)=E_{4}(0,0)$ in green. (For interpretation of the references to colour in this figure legend, the reader is referred to the web version of this article.)

appears also in the harmonic oscillator perturbed by a Dirac delta in both one and three dimensions. This has been shown in [10,42]. For additional information on the Zeldovich effect for Hamiltonians with rapidly decaying potentials and the definition of the Zeldovich spiral, see [62,63].

If the point impurity is centred away from the origin, due to the smooth dependence of the right hand side of (14) on $x_{0}$, the spectral structure of the odd eigenvalues, corresponding to symmetric bound states, does not undergo any major qualitative change. However, the antisymmetric bound states are also affected by the perturbation. As a consequence, the even eigenvalues are no longer equal to those of $H_{0}$, but given instead by functions of the coupling $\lambda$. While the analogue of Fig. 1 is 
$\lambda$

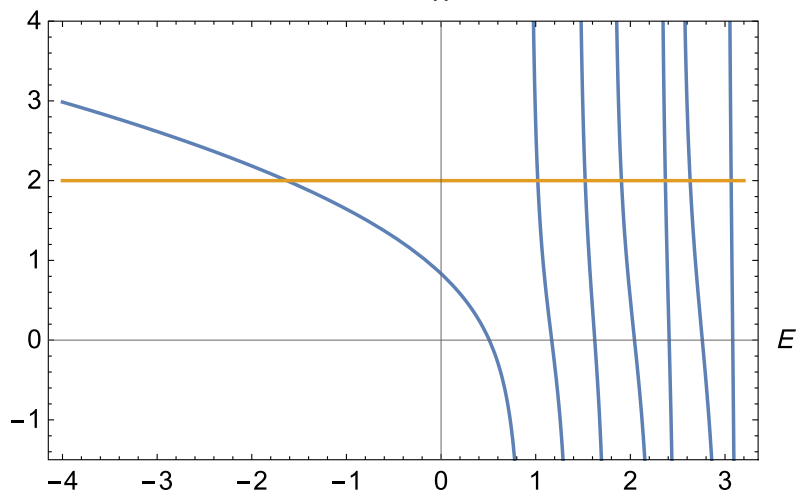

Fig. 3. The $n$th eigenvalue of $H_{\lambda=2, x_{0}=1 / 2}$ is given by the intersection between the $n$th branch of the right hand side of (14) as a function of the energy and the horizontal line $\lambda=2$.

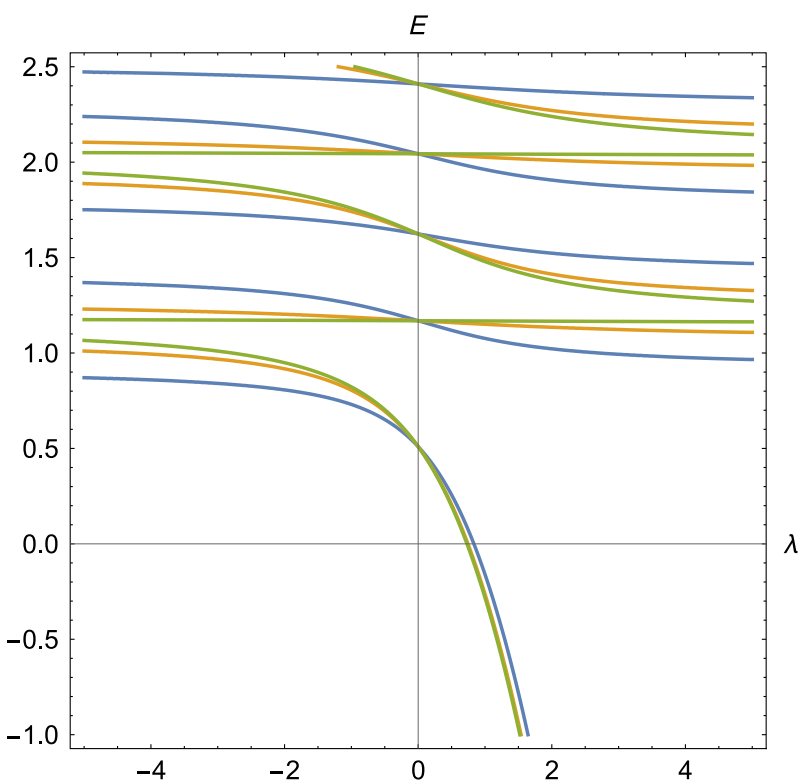

Fig. 4. Plot of the five lowest eigen-energies $E_{n}\left(\lambda, x_{0}\right), n=1, \ldots, 5$, coming from (14), for $x_{0}=0.5$ (blue), 0.2 (yellow), and 0.05 (green). (For interpretation of the references to colour in this figure legend, the reader is referred to the web version of this article.)

plotted in Fig. 3, the plot of the five lowest eigenenergies as functions of $\lambda$ for $x_{0}=0.05,0.2,0.5$ is shown in Fig. 4.

Before proceeding with our discussion, let us note that the attractive Dirac delta interaction at $x_{0}=0$ combined with the $\mathrm{V}$-shaped interaction in one dimension, may be approximated by potentials of the form $\frac{1}{2}|x|-\lambda V_{n}(x)$, where $V_{n}(x)$ is a potential which sinks deeply in a neighbourhood of the origin. By choosing for instance

$$
V_{n}(x)=\frac{n}{3} \frac{e^{-|n x|^{2 / 3}}}{|n x|^{1 / 3}},
$$




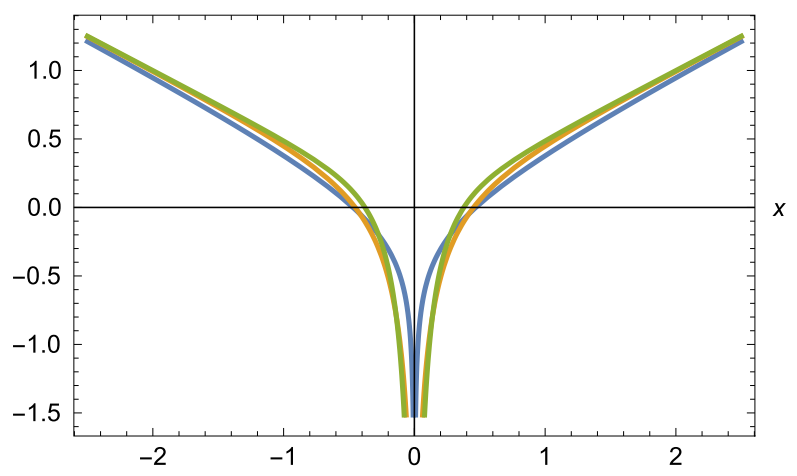

Fig. 5. Plot of the potentials $\frac{1}{2}|x|-V_{n}(x)$ in (16), for $n=1$ (blue), 5 (yellow), and 10 (green). (For interpretation of the references to colour in this figure legend, the reader is referred to the web version of this article.)

we have an example of the aforementioned funnel shaped potential. This example is used in Fig. 5, where we have also chosen $\lambda=1$.

\subsection{The inverse problem: identifying the potential from measured values of the energy}

In this brief subsection, we consider the so-called spectral inverse problem [70], directly related to the operator studied in the previous section. Let us assume that one has two eigenvalues $E_{1}$ and $E_{2}$ and that the potential is a priori known to be $|x| / 2-\lambda \delta\left(x-x_{0}\right)$, one has to determine the values of $\lambda$ and $x_{0}$ so that the resulting Hamiltonian has these two energy levels in the discrete spectrum.

Our motivation can be found in the final section of the paper by Fassari and Inglese on the Hamiltonian of the quantum harmonic oscillator perturbed by a point interaction [33]. An important difference between this latter article and the present one is the following: In [33] it is assumed that $E_{1}$ and $E_{2}$ are the two lowest eigenvalues, as they have used the bound state equations for these particular eigenvalues. They have used the specific integral expressions derived from the so-called Mehler kernel and not a global relation which involves parabolic cylindrical functions. In the present article, we make use of the bound state equation derived from the global expression for the Green function in terms of the Airy function and its derivative.

As shown in the literature on inverse problems [70], an inverse problem is often ill-posed either because it has no solutions in the desired class, or has many (two or more) solutions, or because the solution procedure is unstable, i.e., arbitrarily small errors in the measurement data may lead to indefinitely large errors in the solutions. Consequently, the inverse problem investigated in [33] is ill-posed for this second reason.

Next, we see that an attempt to solve the inverse problem with the a priori assumption that the potential is of the form $|x| / 2-\lambda \delta\left(x-x_{0}\right)$, by means of a global bound state equation, leads to an ill-posed problem having many solutions. In fact using (14), one has just to solve the equations

$$
\frac{A i\left(-2 E_{1}\right) A i^{\prime}\left(-2 E_{1}\right)}{A i\left(-2 E_{2}\right) A i^{\prime}\left(-2 E_{2}\right)}=\frac{A i\left(x_{0}-2 E_{1}\right) A i\left(-x_{0}-2 E_{1}\right)}{A i\left(x_{0}-2 E_{2}\right) A i\left(-x_{0}-2 E_{2}\right)},
$$

and

$$
\lambda=-\frac{A i\left(-2 E_{1}\right) A i^{\prime}\left(-2 E_{1}\right)}{A i\left(x_{0}-2 E_{1}\right) A i\left(-x_{0}-2 E_{1}\right)} .
$$

Eqs. (17) and (18) can be easily solved graphically and, since the Airy functions are oscillatory, will produce infinitely many potentials possessing the given levels. For example, let us choose $x_{0}=1.2557$ and plot in the plane $\left(E_{1}, E_{2}\right)$ the curves solution of (17). Then for every pair of values $E_{1}$ and $E_{2}$, (18) gives the values of $\lambda$. See this in Fig. 6 , where the dotted lines correspond to pairs $\left(E_{1}, E_{2}\right)$ satisfying 


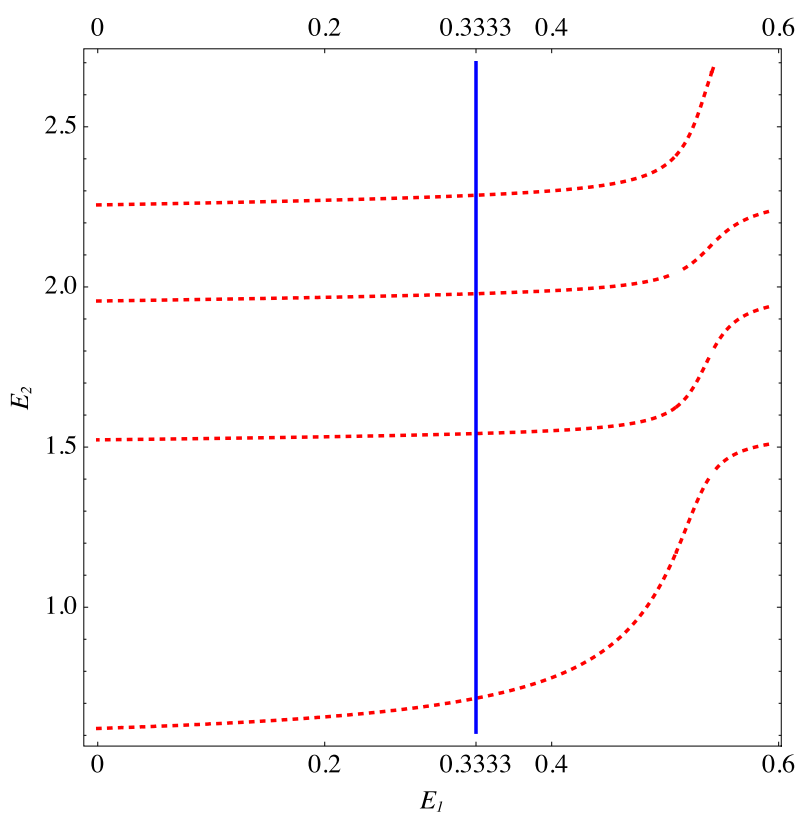

Fig. 6. Plot of the implicit equation (17) for $x_{0}=1.2557$, which has an infinity of branches (dotted red lines). For a fixed value of $E_{1}$, let us say $E_{1}=0.3333$ (blue line), the infinite solutions of the inverse problem are obtained.

(17). If we choose a given value for $E_{1}$, we automatically have all permitted values for $E_{2}$ and $\lambda$. In Fig. 6 , we have chosen $E_{1}=0.3333$. This gives for the first level $E_{2}=0.7158$ with $\lambda=1.3602$. The two next values of $E_{2}$ are $E_{2}=1.5423$ and $E_{2}=1.9791$.

It might also be possible to consider a different type of inverse problem not purely of the spectral type. In addition to the spectral data one might include information related to the eigenfunctions such as the value at a point away from the location of the point impurity or the value of a norming constant, see [71].

\section{The spectrum of $H_{0}$ perturbed by an attractive $\delta^{\prime}$-interaction}

In this section, we analyse the results obtained after the perturbation of $H_{0}$ by two distinct choices of a $\delta^{\prime}$-interaction. We first consider the nonlocal $\delta^{\prime}$-interaction as defined in [11] (see also [1,12,72]). Then, we shall obtain the results after the choice proposed in $[9,13]$.

\subsection{Nonlocal $\delta^{\prime}$-interaction}

In the analysis of this nonlocal $\delta^{\prime}$ perturbation, we shall use similar mathematical techniques as done for the $\delta$ interaction and detailed in [69]. The objective is to find the eigenvalues of the perturbed Hamiltonian as poles of the Green function.

As we have remarked in the Introduction, one of the possible ways to characterise point interactions supported at any point is by giving the matching conditions that wave functions have to satisfy at the given point $[2,73]$. If this point is the origin, the nonlocal $\delta^{\prime}$ interaction is produced by the assignment of the matching conditions (9) for all wave functions $\psi(x)$ :

$$
\psi\left(0^{+}\right)-\psi\left(0^{-}\right)=-\beta \psi^{\prime}(0), \quad \psi^{\prime}\left(0^{+}\right)=\psi^{\prime}\left(0^{-}\right),
$$

where $\psi\left(0^{ \pm}\right)=\lim _{x \rightarrow 0^{ \pm}} \psi(x)$ for any function $\psi(x)$. 
Therefore, if the approach based on the theory of ordinary differential equations were adopted, one should look for those solutions of the differential equation $\frac{1}{2}\left[-\frac{d^{2}}{d x^{2}}+|x|\right] \psi=E \psi$ satisfying the above conditions at the origin. Therefore, while the derivative of the function must be continuous at zero, the function itself must have a jump discontinuity at that point. We remind the reader that, in the absence of a potential, the unique normalised eigenfunction of $-\frac{d^{2}}{d x^{2}} \psi_{\beta}(x)=E \psi_{\beta}(x)$ with the above conditions at the origin is (see [1]):

$$
\psi_{\beta}(x)=\sqrt{\frac{\beta}{8}} \frac{x}{|x|} e^{-\frac{2|x|}{\beta}}, \quad E=-\frac{4}{\beta^{2}} .
$$

If instead one were to repeat the mathematical steps leading to (13), the counterpart of (14) would read

$$
\frac{1}{\tilde{\beta}}-\frac{1}{2} \sum_{n=1}^{\infty} \frac{1}{E_{2 n}-E},
$$

where $E_{2 n}$ are the even energies for $H_{0}$. However, the above series does not converge because the general term of the sequence $\left(E_{2 n}-E\right)^{-1}$ behaves asymptotically like $n^{-2 / 3}$. The cure for this disease is the renormalisation of the coupling constant by introducing an ultraviolet energy cutoff and a counterterm in the reciprocal of the coupling constant, namely:

$$
\frac{1}{\tilde{\beta}(N)}=\frac{1}{\beta}+\frac{1}{2} \sum_{n=1}^{N} \frac{1}{E_{2 n}} .
$$

Although the mathematical procedure leading to the determination of the desired Hamiltonian is rather complicated since it requires the norm resolvent convergence, we can take advantage of the explicit formula of the resolvent of the limiting self-adjoint operator $H_{\beta}$ provided in [69] to get the corresponding expression for the Green's function:

$$
G_{\beta}(x, y ; E)=G_{0}(x, y ; E)+\frac{\Psi(x ; E) \Psi(y ; E)}{\frac{1}{\beta}-\frac{E}{2} \sum_{n=1}^{\infty} \frac{1}{E_{2 n}\left(E_{2 n}-E\right)}} .
$$

The explicit expression of $\Psi(x ; E)$ and further details can be found in [69].

It is crucial now to point out that the series in the denominator of (22) converges, since the general term of the sequence $\left(E_{2 n}\left(E_{2 n}-E\right)\right)^{-1}$ behaves asymptotically like $n^{-4 / 3}$. Then, the values of $E$ giving the poles of the Green function (22) for each value of $\beta$ are given by the following transcendental equation:

$$
\frac{1}{\beta}=\frac{E}{2} \sum_{n=1}^{\infty} \frac{1}{E_{2 n}\left(E_{2 n}-E\right)} .
$$

Similarly to the operations performed for the $\delta$-perturbation, we may write (23) in terms of Airy functions. After some operations (see [69]), we obtain our final result, namely

$$
\beta=\frac{\operatorname{Ai}(0) \operatorname{Ai}(-2 E)}{A i(0) A i^{\prime}(-2 E)-A i^{\prime}(0) A i(-2 E)} .
$$

The odd eigenvalues remain unperturbed under the interaction. As was shown in [61], this means that $A i^{\prime}\left(-2 E_{2 n-1}\right)=0$. The dependence on $\beta$ of the even energy levels is depicted in Fig. 7. The level $E_{0}$ exists for negative values of $\beta$ only. However, the situation is different for all other values of $E_{2 n}$ in terms of $\beta$. There exists a value $\beta_{0}$ such that:

(i) if $\beta<\beta_{0}, E_{2 n-1}<E_{2 n}, n=1,2, \ldots$;

(ii) for $\beta>\beta_{0}$, the situation is the reverse: $E_{2 n-1}>E_{2 n}$.

Crossings occur at $\beta=\beta_{0}$, where $E_{2 n-1}=E_{2 n}$. Therefore, the spectrum of $H_{\beta_{0}}$ is doubly degenerate at all levels. 


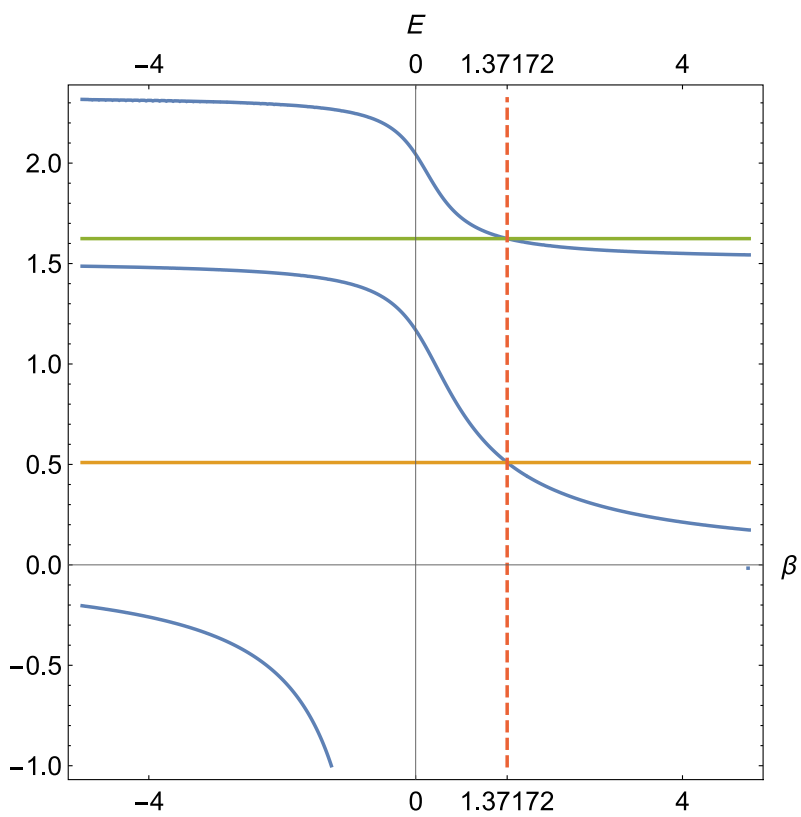

Fig. 7. The level crossings occurring in (23) at $\beta_{0}=1.37172$ between the eigenenergy $E_{2}(\beta)$ (middle blue curve) and $E_{1}(0)$ (orange horizontal line), as well as between the eigenenergy $E_{4}(\beta)$ (upper blue curve) and $E_{3}(0)$ (green horizontal line). (For interpretation of the references to colour in this figure legend, the reader is referred to the web version of this article.)

The value for $\beta_{0}$ is quite easy to find after the remark given in the first sentence right after (23). Since $A i^{\prime}\left(-2 E_{2 n-1}\right)=0$, one finds that

$$
\beta_{0}=-\frac{\operatorname{Ai}(0)}{A i^{\prime}(0)} \approx 1.37172 \text {. }
$$

These results can be compared with those obtained by replacing $H_{0}$ with its counterpart for the one-dimensional harmonic oscillator. The study of the latter has been undertaken in [11,72]. The situation is essentially the same, only numerical values are different. Of course, it is also worth stressing that, while in the case of the harmonic oscillator the length of the interval between two adjacent energy levels is constant, that no longer holds in our model (see [61]). In particular, level crossings in the model with the harmonic confinement occur for $\beta_{0} \approx 1.47934$.

Fig. 7 shows some other interesting facts. For instance, we see that the curves representing the unperturbed odd energy levels do not serve as horizontal asymptotes for the curves representing the even levels. This is in sharp contrast to the situation shown in Fig. 2. This fact confirms the extremely singular nature of the nonlocal $\delta^{\prime}$ interaction which, in physical terms, produces a spectral effect far stronger than the aforementioned one named after Zeldovich.

In analogy with the behaviour observed in $[11,72]$ for the harmonic oscillator perturbed by the $\delta^{\prime}-$ interaction, the symmetry of the wave function changes from symmetric to antisymmetric crossing the point $\beta=\beta_{0}$. Therefore, this is evidence that a quantum phase transition has occurred.

As a remark, it might be worth pointing out that the level crossings observed in the spectrum of the three-dimensional isotropic harmonic oscillator perturbed by a $\delta$-interaction occur at the point

$$
\beta_{0}=\frac{\pi \Gamma(1 / 4)}{\Gamma(3 / 4)} \approx 9.29495,
$$

see $[11,40,42,43]$. There is, however, a big qualitative difference: for $\beta>\beta_{0}$ the symmetric bound state energy $E_{2 n}(\beta)$ falls below the unperturbed antisymmetric bound state energy $E_{2 n-1}=\frac{3}{2}+$ 
$(2 n-1)$, so that the symmetry properties in three dimensions are reversed with respect to the symmetry properties in one dimension.

\subsection{Local $\delta^{\prime}$-interaction}

In order to illustrate the sensitivity of these calculations to the precise interpretation of highly singular point potentials we shall examine the effect of the potential $V(x)=-\lambda \delta(x)+\mu \delta^{\prime}(x)$ on the conical oscillator when interpreted, as is frequently done, as the set of boundary conditions [9].

It is noteworthy that, while the nonlocal $\delta^{\prime}$ interaction is not compatible with a Dirac delta interaction $-\lambda \delta(x)$ due to the incompatibility of the matching conditions defining each one, this is not the case for the local $\delta^{\prime}$ interaction. In fact, a potential of the form $V(x)=-\lambda \delta(x)+\mu \delta^{\prime}(x)$ may be defined through matching conditions (10). In this case, $\mu \delta^{\prime}(x)$ has a local character. Then, the total Hamiltonian $H=H_{0}-\lambda \delta(x)+\mu \delta^{\prime}(x)$ is self-adjoint on a domain of functions showing a discontinuity at the origin, so that the product of each function $\psi(x)$ in this domain is given by, respectively:

$$
\delta(x) \psi(x)=\frac{\psi\left(0^{+}\right)+\psi\left(0^{-}\right)}{2} \delta(x)
$$

and

$$
\delta^{\prime}(x) \psi(x)=\frac{\psi\left(0^{+}\right)+\psi\left(0^{-}\right)}{2} \delta^{\prime}(x)-\frac{\psi^{\prime}\left(0^{+}\right)+\psi^{\prime}\left(0^{-}\right)}{2} \delta(x) .
$$

A slight generalisation of these products was given in [74,75].

The previous analysis relative to the characterisation of the resolvent of the operator $H_{0}-\beta\left|\delta^{\prime}\right\rangle\left\langle\delta^{\prime}\right|$ for the nonlocal $\delta^{\prime}$ is not straightforwardly applicable here. Instead, we use a technique that has been discussed in [9], which relies on calculations with the Green's functions of $H_{0}$ and $H$. In fact, if $G_{0}\left(x, x^{\prime}, E\right)$ is the Green function of $H_{0}$, then bound states $\psi(x)$ with energy $E$ corresponding to the total Hamiltonian $H=H_{0}-\lambda \delta(x)+\mu \delta^{\prime}(x)$ as above have the following form:

$$
\psi(x)=\int G_{0}\left(x, x^{\prime}, E\right)\left[-\lambda \delta\left(x^{\prime}\right)+\mu \delta^{\prime}\left(x^{\prime}\right)\right] \psi\left(x^{\prime}\right) d x^{\prime} .
$$

This expression yields a homogeneous system of four equations with four unknowns. In order to obtain nontrivial solutions, the determinant of the system has to be equal to zero. This determinant can be further simplified so as to obtain the following expression (see [9]):

$$
\left|\begin{array}{ccc}
2 & -1-\mu & 0 \\
-1 & 1+\frac{\lambda}{2} A+\frac{\mu}{2} & \frac{\mu}{2} A \\
0 & -\frac{\mu}{A} & 1
\end{array}\right|=1+\lambda A+\mu^{2}=0 .
$$

Then, the energy levels of the total Hamiltonian $H$ are the roots of the above determinant. Here, $A$ is related to the Green function $G_{0}\left(x, x^{\prime} ; E\right)$ defined in (12) by

$$
A=G_{0}\left(0^{+}, 0 ; E\right)=\frac{A i(-2 E)}{A i^{\prime}(-2 E)} .
$$

Therefore, the vanishing of the determinant (29) reduces to

$$
\frac{A i^{\prime}(-2 E)}{A i(-2 E)}=-\frac{\lambda}{\mu^{2}+1} \text {. }
$$

Hence, if $\lambda=0$ there are no new states, and if $\mu=0$ the new states coincide with those of (15). In Fig. 8 we plot the structure of the first five energy levels for this Hamiltonian for a particular value of $\lambda$, as functions of $\mu$. 


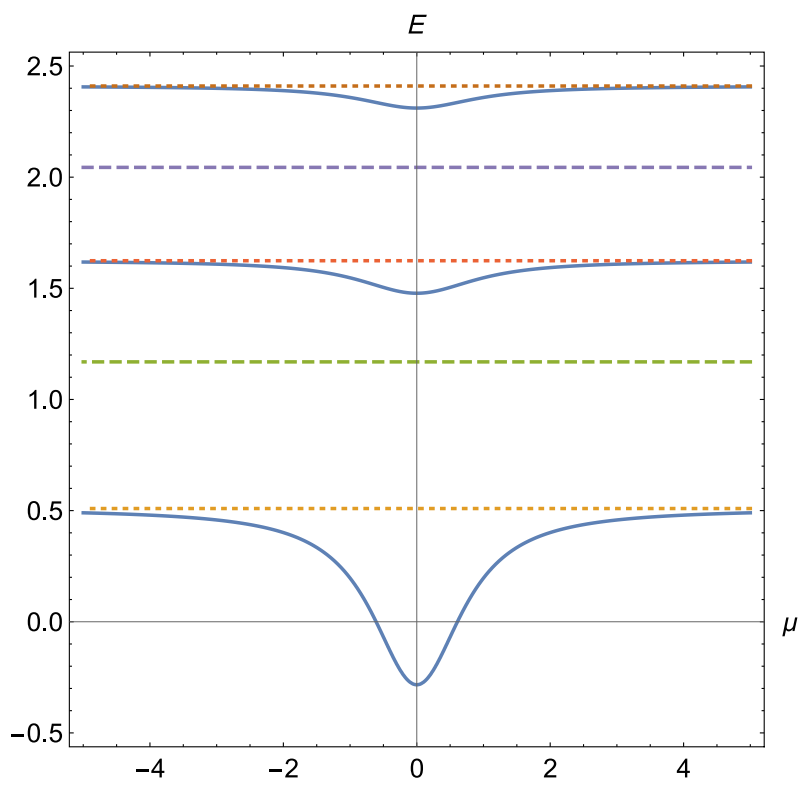

Fig. 8. The first five energy levels of the conic potential plus the perturbation $V(x)=-\lambda \delta(x)+\mu \delta^{\prime}(x)$ for $\lambda=1$ as functions of $\mu$, coming from (31): in blue the symmetric levels and dashed the antisymmetric ones (they do not depend on $\mu$ ). The dotted lines correspond to the symmetric energy levels of the unperturbed conic potential.

\section{Concluding remarks}

We have perturbed the Hamiltonian with a V-shaped potential $H_{0}=\frac{1}{2}\left[-\frac{d^{2}}{d x^{2}}+|x|\right]$ by various point interactions. The operator $H_{0}$ describes a quantum oscillator with a conical confinement instead of the usual harmonic confinement. The point interactions studied here are of three basic types: Dirac delta $\delta$, nonlocal $\delta^{\prime}$ and finally local $\delta^{\prime}$. In all three cases, the model is solvable in the sense that we are able to determine the perturbed energy levels in terms of the interaction parameters. We have obtained these energy levels as poles of the resolvent of the perturbed Hamiltonian. These poles should depend on the given parameters, something which is not true in all cases. Our results do not differ qualitatively from those previously obtained when $H_{0}$ is instead the Hamiltonian of the harmonic oscillator. Our study of these models was motivated by the increasing use of the V-shaped potential in condensed matter physics.

Let us summarise our results as follows:

(i) When $H_{0}$ undergoes a perturbation of the type $-\lambda \delta(x)$, even energy levels, pertaining to antisymmetric bound states, are unaffected by the perturbation. This is not the case for odd energy levels, pertaining to symmetric bound states, for which the energy decreases as $\lambda$ increases. For odd energy levels excluding the first one, the function of the energy $E$ in terms of $\lambda$ shows an asymptotic behaviour towards the upper (lower) even energy value as $\lambda \rightarrow-\infty$ $(\lambda \rightarrow \infty)$. The first energy level shows the same behaviour for $\lambda \rightarrow-\infty$, while the value of the energy goes to $-\infty$ as $\lambda \rightarrow 0^{-}$.

(ii) However, when the perturbation is of the type $-\lambda \delta\left(x-x_{0}\right)$, even levels are no longer constant with respect to $\lambda$, but instead undergo a change, which is usually small. All levels decrease as $\lambda$ increases and the energy of the first one still goes to $-\infty$ as $\lambda \rightarrow \infty$.

(iii) When the perturbation is of the type $\delta^{\prime}$ the situation changes dramatically. First of all, we consider two types of $\delta^{\prime}$ interactions: nonlocal and local. We have constructed the resolvent operator for the Hamiltonian $H_{0}$ decorated with a nonlocal $\delta^{\prime}$ perturbation centred at the origin: 
$H_{\beta}=H_{0}-\beta\left|\delta^{\prime}\right\rangle\left\langle\delta^{\prime}\right|$. Contrary to the case of the Dirac delta perturbation, this construction requires the renormalisation of the coupling parameter. Odd energy levels, pertaining to symmetric bound states, are unchanged with respect to the renormalised form factor $\beta$, but even energy levels, which correspond to antisymmetric bound states, decrease as $\beta \rightarrow \infty$. At a particular value of $\beta$, which is the same for all levels $\beta \approx 1.37172$, we observe a noteworthy phenomenon: the existence of level crossings. The energy of each even level becomes lower than the energy of the previous odd level (except the first one) and remains lower as $\beta \rightarrow \infty$.

(iv) When the perturbation of type $\delta^{\prime}$ is local, it is possible to combine it with a Dirac delta perturbation, so that the total Hamiltonian becomes $H=H_{0}-\lambda \delta(x)+\mu \delta^{\prime}(x)$ in this case. We have investigated the energy levels of $H$ using its Green function. While the energy of even levels remains unaffected, the odd levels are lowered but level crossings are absent, provided that $\lambda \neq 0$. If $\lambda=0$, none of the energy values is affected by the $\mu \delta^{\prime}(x)$ term.

We believe the current findings pave the way to the investigation of both the conic and the pyramidal oscillator perturbed by a point impurity in two and three dimensions. Furthermore, if our Hamiltonian were added to the two-dimensional negative Laplacian, it could provide a model for a V-shaped quantum well (heterostructure in which electrons can move freely in two dimensions but are confined in the third one). We are also convinced that it should be possible to show that the selfadjoint operator $H_{\beta}$ whose Green's function, given by (22), has been obtained by renormalising the coupling constant can also be obtained by means of the so-called Cheon-Shigehara approximation involving a suitable triple of Dirac distributions, given that such a result holds when the free Hamiltonian is the one of the harmonic oscillator (see [12,72] and the references therein). The latter approximation, in turn, grants the possibility of approximating the nonlocal $\delta^{\prime}$-interaction by means of a combination of three sharply peaked attractive potentials with shrinking support, as was achieved in the absence of confinement in [76].

\section{Acknowledgements}

Partial financial support is acknowledged to the Spanish Junta de Castilla y León and FEDER (Project VA057U16) and MINECO (Project MTM2014-57129-C2-1-P). We are indebted to G. Inglese for an illuminating discussion regarding the inverse problem dealt with in Section 2.1. S. Fassari and M.L. Glasser also wish to thank the entire staff at Departamento de Física Teórica, Atómica y Óptica, Universidad de Valladolid, for their warm hospitality throughout their stays.

\section{References}

[1] S. Albeverio, F. Gesztesy, R. Høegh-Krohn, H. Holden, Solvable Models in Quantum Mechanics, second ed., in: (AMS Chelsea Series), American Mathematical Society, Providence, RI, 2004.

[2] S. Albeverio, P. Kurasov, Singular Perturbations of Differential Operators (Cambridge UK, 2000).

[3] A.V. Zolotaryuk, Y. Zolotaryuk, J. Phys. A 48 (2015) 035302.

[4] A.V. Zolotaryuk, Y. Zolotaryuk, Phys. Lett. A 379 (2015) 511-517.

[5] M. Gadella, F.J.H. Heras, J. Negro, L.M. Nieto, J. Phys. A 42 (2009) 465207.

[6] S. Albeverio, S. Fassari, F. Rinaldi, J. Phys. A 48 (2015) 185301.

[7] F. Erman, M. Gadella, H. Uncu, Phys. Rev. D 95 (2017) 045004.

[8] M.H. Al-Hashimi, A.M. Shalaby, U.J. Wiese, Phys. Rev. D 89 (2014) 125023.

[9] M. Gadella, M.L. Glasser, L.M. Nieto, Internat. J. Theoret. Phys. 50 (2011) 2144-2152.

[10] S. Fassari, F. Rinaldi, Rep. Math. Phys. 69 (2012) 353-370.

[11] S. Albeverio, S. Fassari, F. Rinaldi, J. Phys. A 46 (2013) 385305.

[12] S. Fassari, F. Rinaldi, Rep. Math. Phys. 64 (2009) 367-393.

[13] M. Gadella, M.L. Glasser, L.M. Nieto, Internat. J. Theoret. Phys. 50 (2011) 2191-2200.

[14] M. Gadella, M.A. García-Ferrero, S. González-Martín, F.H. Maldonado-Villamizar, Internat. J. Theoret. Phys. 53 (2014) 1614-1627.

[15] X. Wang, L.H. Tang, R.L. Wu, N. Wang, Q.H. Liu, Commun. Theor. Phys. 53 (2010) 247-249.

[16] M.G. Espinosa, P. Kielanowski, J. Phys: Conf. Ser. 128 (2008) 012037.

[17] J.J. Alvarez, M. Gadella, L.P. Lara, F.H. Maldonado-Villamizar, Phys. Lett. A 337 (2013) 2510-2519.

[18] W. Häusler, B. Kramer, Phys. Rev. B 47 (1993) 16353-16357.

[19] S. Kvaal, M. Hjorth-Jensen, H. Møll Nilsen, Phys. Rev. B 76 (2007) 085421.

[20] S. Kvaal, Phys. Rev. B 80 (2009) 045321. 
[21] J.M. Muñoz Castaneda, J. Mateos Guilarte, A.M. Mosquera, Phys. Rev. D 87 (2013) 105020.

[22] M. Asorey, D. Garcia-Alvarez, J.M. Muñoz Castañeda, J. Phys. A 39 (2006) 6127-6136.

[23] M. Asorey, J.M. Muñoz Castañeda, Nuclear Phys. B 874 (2013) 852-876.

[24] J.M. Muñoz Castañeda, K. Kirsten, M. Bordag, Lett. Math. Phys. 105 (2015) 523-549.

[25] J. Mateos Guilarte, J.M. Muñoz Castañeda, Internat. J. Theoret. Phys. 50 (2011) 2227-2241.

[26] M. Bordag, J.M. Muñoz Castañeda, J. Phys. A 45 (2012) 374012.

[27] G. Barton, J. Phys. A: Math. Gen. 37 (2004) 1011-1049.

[28] M. Bordag, Phys. Rev. D 89 (2014) 125015.

[29] H. Uncu, D. Tarhan, Ö.E. Müstecaplığlu, Phys. Rev. A 76 (2007) 013618.

[30] P. Shea, B.P. Van Zyl, R.K. Bhaduri, Amer. J. Phys. 77 (2009) 511-515.

[31] J. Goold, D. O’Donoghue, Th. Busch, J. Phys. B 41 (2008) 215301.

[32] S. Fassari, G. Inglese, Helv. Phys. Acta 67 (1994) 650-659.

[33] S. Fassari, G. Inglese, Helv. Phys. Acta 70 (1997) 858-865.

[34] M.P. Avakian, G.S. Pogosyan, A.N. Sissakian, V.M. Ter-Antonyan, Phys. Lett. A 124 (1987) 233-236.

[35] V. Lutsenko, L.G. Mardoyan, G.S. Pogosyan, A.N. Sissakian, V.M. Ter-Antonyan, J. Phys. A: Math. Gen. 22 (1989) $2739-2749$.

[36] E. Demiralp, J. Phys. A: Math. Gen. 22 (2005) 4783-4793.

[37] E. Demiralp, Czech. J. Phys. 55 (2005) 1081-1084.

[38] M. Correggi, G. Dell'Antonio, D. Finco, J. Funct. Anal. 255 (2008) 502-531.

[39] T.A. Filatova, A.I. Shafarevich, Theoret. Math. Phys. 164 (2010) 1064-1080.

[40] S. Fassari, G. Inglese, Helv. Phys. Acta 69 (1996) 130-140.

[41] J. Brüning, V. Geyler, I. Lobanov, J. Math. Phys. 45 (2004) 1267-1290.

[42] S. Albeverio, S. Fassari, F. Rinaldi, Nanosyst.: Phys. Chem. Math. 7 (5) (2016) 803-815.

[43] S. Albeverio, S. Fassari, F. Rinaldi, Nanosyst.: Phys. Chem. Math. 7 (2)(2016) 268-289.

[44] P. Harrison, Quantum Wells Wires Dots, third ed., in: Theoretical and Computational Physics of Semiconductor Nanostructures, Wiley, Chichester, West Sussex, UK, 2009.

[45] E.F. Schubert, B. Ullrich, T.D. Harris, J.E. Cunningham, Phys. Rev. B 38 (12) (1988) 8305-8309.

[46] E. Kasapoglu, H. Sari, I. Sokmen, Physica E 19 (2003) 332-335.

[47] E. Kasapoglu, I. Sokmen, Physica E 27 (2005) 198-203.

[48] K. Nozari, M. Madadi, Phys. Scr. 69 (2004) 244-248.

[49] T.B. Boykin, G. Klimeck, P. von Allmen, S. Lee, F. Oyafuso, J. Appl. Phys. 97 (11) (2005) 113702.

[50] D. Brinkmann, A. Löffler, G. Fishman, J. Phys. I France 7 (1997) 1221-1231.

[51] R. Horvat, D. Kekez, D. Klabucar, D. Palle, Phys. Rev. D 44 (5) (1991) 1585-1590.

[52] R. Horvat, D. Kekez, D. Palle, D. Klabucar, Z. Phys. C 68 (1995) 303-313.

[53] O. Mustafa, Czech. J. Phys. 54 (5) (2004) 529-542.

[54] L. Yan, G.-Y. Xu, Y.-J. Wang, X.-F. Liu, J.-R. Hang, Commun. Theor. Phys. 52 (2009) 431-434.

[55] G.-Y. Xu, et al., Chin. Phys. B 17 (11) (2008) 4158-4162.

[56] D.P. Foty, Cryogenics 30 (1990) 1056-1063.

[57] R.J. Lange, J. Math. Phys. 56 (12) (2015) 122105.

[58] S. López, F. Domínguez-Adame, Semicond. Sci. Technol. 17 (2002) 227-229.

[59] R.P.A. Lima, M. Amado, F. Domínguez-Adame, Nanotechnology 19 (2008) 135402.

[60] C. González-Santander, F. Domínguez-Adame, Phys. Lett. A 374 (2010) 2259-2261.

[61] J. Lörinczi, J. Małecki, J. Differential Equations 253 (2012) 2846-2871.

[62] M. Combescure, et al., Internat. J. Modern Phys. B 21 (2007) 3765.

[63] A. Farrell, Z. MacDonald, B. van Zyl, J. Phys. A 45 (2012) 045303.

[64] P. Şeba, Czec. J. Phys. 36 (1986) 667-673.

[65] M. Gadella, J. Negro, L.M. Nieto, Phys. Lett. A 373 (2009) 1310-1313.

[66] M. Gadella, J. Mateos-Guilarte, J.M. Muñoz Castañeda, L.M. Nieto, J. Phys. A 49 (2016) 015204.

[67] Y.D. Golovaty, Methods Funct. Anal. Topology 18 (2012) 243-255.

[68] M.L. Glasser, L.M. Nieto, Can. J. Phys. 93 (2015) 1588-1596.

[69] S. Fassari, M. Gadella, M.L. Glasser, L.M. Nieto, 2017. arXiv:1706.04916v1 [math-ph].

[70] S.I. Kabanikhin, J. Inverse Ill-Posed Probl. 16 (2008) 317-357.

[71] J. Eckardt, G. Teschl, Trans. Amer. Math. Soc. 365 (7) (2013) 3923-3942.

[72] S. Albeverio, S. Fassari, F. Rinaldi, J. Phys. A 49 (2016) 025302.

[73] P. Kurasov, J. Math. Ann. Appl. 201 (1996) 297-323.

[74] A.V. Zolotaryuk, Phys. Lett. A 374 (2010) 1636-1641.

[75] F. Maldonado-Villamizar, Phys. Scr. 90 (2015) 065202

[76] P. Exner, H. Neidhardt, V.A. Zagrebnov, Comm. Math. Phys. 224 (2001) 593-612. 